\title{
Simulation Study of Zigbee and Enocean Home Automation Standards
}

\author{
Sukesha ${ }^{1}$, Harneet Kaur ${ }^{2}$ \\ Assistant professor, Dept of IT, UIET, Panjab University, Chandigarh, India ${ }^{1}$ \\ PG Student, Dept of IT, UIET, Panjab University, Chandigarh, India ${ }^{2}$
}

\begin{abstract}
The major design challenge of home automation is to choose best standard for controlling devices in existing home environments without any changes in infrastructure. In previous years Zigbee was the promising technology for home automation. But now a day's Enocean is the new standard which competes with Zigbee. The temperature of the house is hard and complex to understand since a lot of factors has to be considered. Furthermore, the quality of the house including temperature control not only affects the house performance it also affects the human comfort in their house. In this paper temperature of the house is controlled by using Zigbee and Enocean because of their some features like low cost, low power usage and mesh networking. The work presented here is to show how we can implement Zigbee and Enocean model by using Matlab/Simulink. Some parameters of these technologies are also compared such as: delay, energy per packet and received signal strength indicator (RSSI).
\end{abstract}

KEYWORDS: Home automation, Zigbee, Enocean, delay, energy per packet, received signal strength indicator (RSSI).

\section{I.INTRODUCTION}

In the past decade home automation was possible with limited distance because signals could travel through precise network due to phase couplers or wired connections between two devices. Wireless devices increase the system reliability in large networks. Therefore wireless is the new solution for large homes and power line issues. The primary goal of wireless technology is to reduce installation cost and labour work [1]. Devices which are used in homes for automation are battery powered and battery less with low power Radio Frequency (RF). RF technology has a biggest advantage over infrared that new device can easily be added and removed. Creation of network is the basic requirement for automating home. Wireless home automation networks (WHANs) enable monitoring and control appliances or devices for home user comfort and efficient home management. After forming a network there are certain events that take place like manual events, triggered events and timed events. Home automation systems form single stand alone devices for full house systems where every device or appliance can be controlled in the Home Automation Network (HAN).

Home automation transmission channels: Data can be transmitted through wired or wireless connections between devices in any electric system. Wired methods to transmit the data include structured wiring, coaxial cable, optical cable, phone line and power line. Furthermore wireless transmission channel include radio frequency (RF) technology as well as infrared (IR) technology. Structured wiring, power line and radio frequency are the common techniques used in home automation, which are discussed here briefly:

A. Structured wiring: Structured wiring is very common type of transmission in home automation systems. Twisted pair has two insulated conductors that are symmetrically twisted around their common axis. The twisting reduces disturbance currents, electric and magnetic field.

B. Power-Line: Power line communication is a technology in which data is send over existing wires. This is the major difference that it does not need extra wiring. It works as a half duplex manner. It is broadly divided into narrowband signals and broadband signals. Narrowband signals works at low frequencies whereas broadband signals works at high frequencies [2]. 


\section{? \\ International Journal of Advanced Research in Electrical, Electronics and Instrumentation Engineering}

(An ISO 3297: 2007 Certified Organization)

Vol. 3, Issue 9, September 2014

C. Radio Frequency $(R F)$ : Wireless Radio Frequency channel data is sent wirelessly without cables in home automation systems. Drawback of Infrared is that data should be sent in line of sight (LOS) and up to short distance. Which is overcome by RF technology. RF signals attenuate quickly and only pass through one or two walls in a house.

Home automation is the residential extension of building automation in all the devices are linked together and these devices are monitored or controlled through intelligent network over a medium such as coaxial cable, power line, radio frequency or by a spread spectrum technology by a coordinator or computer. Home automation also refers to the use of computer or information technology to control home appliances.

\section{Types of home automation}

A. Energy management and climate control system: Unnecessary wastage of energy can be avoided through energy management system. In which energy is controlled by several types of sensors that monitor parameters such as temperature, humidity, light, and presence. For example when sunlight enters the room through windows or doors then electrical lightning can be dimmed or turned off by collecting a command from photo sensors. Energy can also be conserved through climate control system by reducing the demands of heating or cooling that also increase comfort [34].

B. Lightning, windows and appliance control system: Lights of home can be controlled in many different ways like can be controlled by a single switch which reduces the wire connections or can be controlled by obtaining a command from remote controller. Furthermore they automatically turned on through sensors when they detect the presence of person in a room.

C. Healthcare systems and home monitoring: The symptoms of disable person include chronic disease like cancer, diabetes, mental impairment, birth defects, malnutrition and HIV/AIDS. It is not possible to take care of all these patients in hospital or nursing homes for uncertain period of time. Its solution is to provide healthcare services or assistive services at their own homes. Home automation plays a vital role for elderly and disabled person, who remain at home by wearable sensors that can periodically reports the several body parameters such as temperature, blood pressure, and insulin [5].

D. Security and access control systems: Security and safety can be provided by several sensors like smoke sensors, glass break sensors (can be distributed inside or outside of house) and motion sensors. These sensors when detect risk situations and trigger an appropriate action. For example smoke sensor may turn the fire alarm on. Similar detection system can also be used to detect rain and water level to prevent floods or other disasters. Security can also be provided by access control systems in which person's identity such as facial recognition, finger prints or any password can be checked before providing entry to a room.

E. Audio visual and entertainment systems: In smart homes audios and videos can be controlled. Whenever any visitor enters the house then one can turn on $\mathrm{T} \mathrm{V}$ or turn on stereo and voice can be recognized.

\section{IMPLEMENTATION OF WIRELESS STANDARDS IN MATLAB/SIMULINK}

\section{A. Zigbee}

The new short range, low power, low rate wireless networking protocol, Zigbee, complements the high data rate technologies such as WLAN and open the door for many new applications. This standard operates at three bands, the $2.4 \mathrm{GHz}$ band with a maximum rate of $250 \mathrm{kbps}$, the $915 \mathrm{MHz}$ band with a data rate of $40 \mathrm{kbps}$, and the $868 \mathrm{MHz}$ band with a data rate of $20 \mathrm{kbps}$ [1,6-7]. Zigbee capable sensors can be used in turning On/Off the AC if the temperature exceeded a certain value, turning On/Off the lights and locking doors. In the military field, sensors running Zigbee can be used to observe and track the movement of the enemy. Zigbee protocol stack composed of four main layers: Physical layer, Medium access control (MAC) layer, Network layer and application layer.

\section{B. Enocean}

The Enocean technology is an energy harvesting wireless technology used primarily in building automation systems. This is also applied to other applications in industry, transportation and smart homes. Enocean standard operates with ultra low power consumption and enables wireless communications between battery less wireless sensors, switches, 


\title{
International Journal of Advanced Research in Electrical, Electronics and Instrumentation Engineering
}

\author{
(An ISO 3297: 2007 Certified Organization)
}

\section{Vol. 3, Issue 9, September 2014}

controllers and gateways. In March 2012, the Enocean wireless standard was ratified as the international standard ISO/IEC 14543-3-10. Enocean protocol stack composed of four main layers: physical layer, data link layer and network layer. On physical layer secured data is transmitted by using ASK on either $315 \mathrm{MHz}$ or $868 \mathrm{mMHz}$ with data rate $125 \mathrm{~kb} / \mathrm{s}$. The main goal of Enocean is energy harvesting with extremely ultra-low power consumption. The main advantage of this standard it is easy to install no wires are required and time is saved [8-10].

C. Implementation of Zigbee Model

Zigbee is a light weight IEEE 802.15.4 standard based technology for low data rate, low power consumption, reliability, and short range applications. The Implementation was built in Matlab/Simulink using fundamental components in Simulink to demonstrate how reliably complex modulation schemes can be built, cost effectively and efficiently. The design of Zigbee model for both transmitter and receiver using OQPSK modulation scheme is shown in Fig. 1 given below.

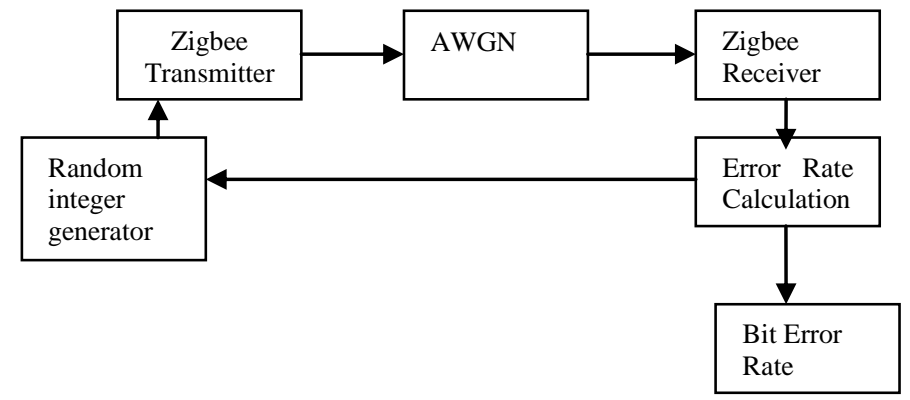

Fig.1 Block diagram for Zigbee [11]

This model includes the following major building blocks: a transmitter which uses OQPSK modulator, a receiver which uses OQPSK demodulator, and an Additive White Gaussian Noise (AWGN) channel. For the 2.4 GHZ model, a random integer generator block generates a number randomly between 1 and 16. Then, this integer is taken as input to the transmitter, which transmits it into32 bits. The 32-bit-stream is taken as an input to the OQPSK modulation block. After modulation, noise is added to the modulated stream using the AWGN block. The latter is then passed through the OQPSK demodulation block before being received. The BER of the received data is calculated as follow: The received 32 bits are sent to the demodulator which converts them back to an integer. Then, the integer-to-bit converter converts the received integer to a 4-bit-stream.Finally, the 4-bit-stream is compared with the original one and the BER is calculated.

Transmitter: On the transmitter, spreading is done using a PN sequence then the spreading bits are modulated using OQPSK modulation. Step by Step the Functions of Zigbee transmitter are given below

a. Generating binary data stream: By using Random integer generator block in the communication toolbox. We can generate binary data stream of $250 \mathrm{~kb} / \mathrm{s}$. In a real time scenario, this data stream is supplied by application that will generate information to be transmitted.

a. $\quad$ Generating PN sequence: 32 bit PN sequence of $2 \mathrm{Mbps}$ data rate is generated using $\mathrm{PN}$ sequence generator block in communication tool box. 32 bit Pseudo Noise code is achieved by adjusting the parameters like generator polynomial, initial states, sample time and output data type, we can achieve

b. Generating DSSS signal: After generating the input bit stream and PN sequence, the generated code is multiplied to get direct spread spectrum signal (DSSS).

Receiver: On the receiver side, OQPSK is done and then the spreaded bits are despreaded using PN chip sequence. Step by step the functions of Zigbee receiver are given below: 


\title{
International Journal of Advanced Research in Electrical, Electronics and Instrumentation Engineering
}

\author{
(An ISO 3297: 2007 Certified Organization)
}

\section{Vol. 3, Issue 9, September 2014}

The transmitted signal is passed through a AWGN channel. The noisy version of the transmitted signal at the input if receiver is received

b. Delay of one bit period is introduced in the transmitter at quadrature side and then same amount of delay is produced on the receiver side.

D. Implementation of Enocean model

Enocean model is implemented in the Matlab/Simulink like Zigbee. Enocean devices usually repeat messages several times in sub telegrams with a random back-off to reduce transmission and collision errors. Model of Enocean is shown in Fig. 2

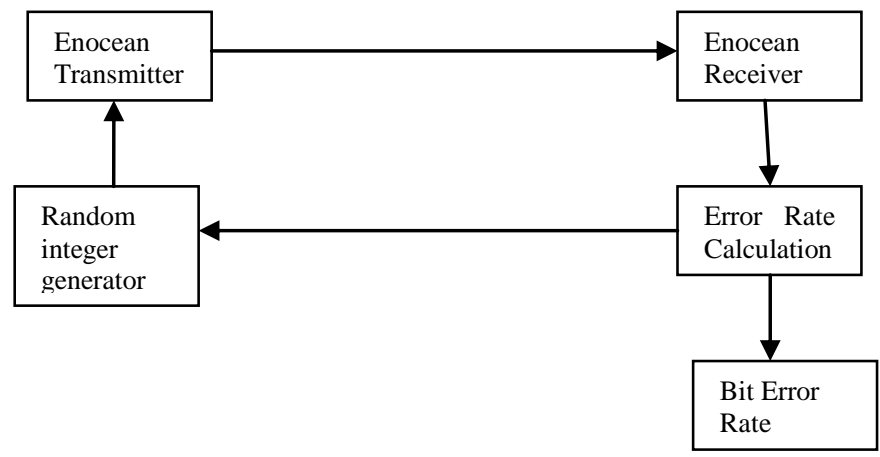

Fig. 2 Block diagram for Enocean standard

For Enocean same model is created as Zigbee. In this Model the input stream is having the data rate of $125 \mathrm{~kb} / \mathrm{s}$. First two subsystems are taken in which Enocean transmitter and receiver are created. Transmitter receives the input from random integer generator block which is present in the communication toolbox then passes this information to the Enocean receiver to control the temperature of the house. In this model AWGN is not used because here noise is internally removed before passing it to the receiver. Here One box is used i.e. bit error rate to count the number of errors in transmitted and received bits.

\section{IMPLEMENTATION OF BASIC MODEL FOR HOUSE}

In Fig. 3 there is one house that containing five rooms including kitchen bathroom and one hallway. In this house only one temperature sensor is used to control the temperature of the whole house rather than single room. First, the temperature of the house is detected by the sensor then input from the house is given to the controller as a heat to control the temperature. There are two switches temperature switch and manual switch. . In temperature switch there are two strokes on and off whereas manual switch is optional. Controller receives the input from sensor which is controlled by the Zigbee standard and Enocean standard in separate models. Sensor only receives the input from the house whereas controller plays the role of both controlling and receiving. It controls the input received from the sensor. It also receives the input from each room of the house and then controls it. In control part when sensors detect that the temperature of the house is higher than the threshold value which sets for the temperature of the house then AC automatically turns ON. Similarly when the temperature of the house is lower than the threshold value then it automatically turns OFF. Output received from the control part is given to the GOTO command. This is used to transfer the execution from one place to another place. Bus selector is used to receive the output from the house and to produce it in the form of graphs. 


\section{International Journal of Advanced Research in Electrical, Electronics and Instrumentation Engineering}

(An ISO 3297: 2007 Certified Organization)

Vol. 3, Issue 9, September 2014

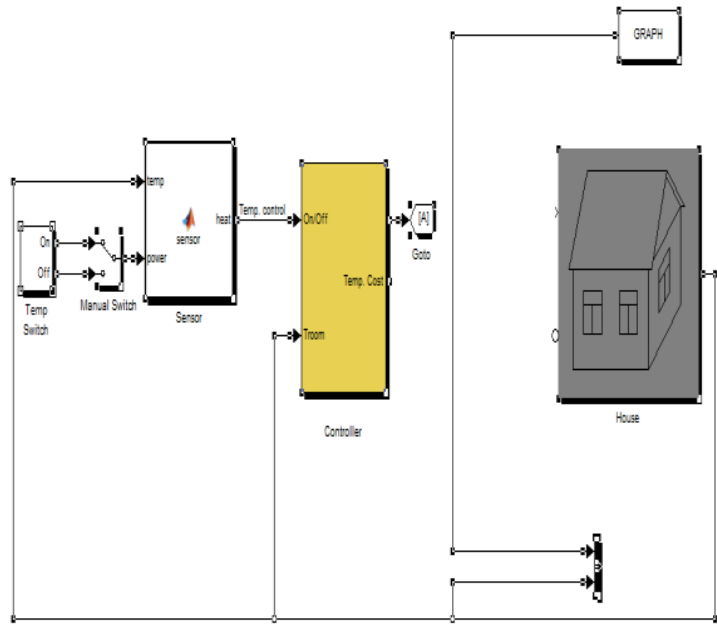

Fig. 3 Model for house in Matlab/Simulink

\section{SIMULATION RESULTS AND ANALYSIS}

This section discusses the results for Zigbee and Enocean by controlling the temperature of the house. For this purpose Zigbee and Enocean models are created in Matlab/Simulink software for wireless communication between devices. Further the basic model for house is created in Matlab/Simulink software in which these two technologies are embedded in house. Zigbee and Enocean are used to control the temperature of the house. Performance of these standards is compared by taking several parameters such as energy per packet, delay, and received signal strength indicator (RSSI). The performance of home automation wireless standards is checked according to different parameters. The results of the simulation study are plotted as graphs and conclusions are drawn on the basis of these graphs. For this following parameters are considered:

- Delay: Time taken for a data packet to reach its destination node. It also includes the delay caused by route discovery process and queue in data packet transmission. From theoretical point of view the transmission delay can be written as [12]

$$
D=\frac{L}{R b}+T_{\text {Prop }}+T_{\text {Proc }}
$$

Where, $\mathrm{T}_{\text {prop }}$ is the Propagation delay, $\mathrm{T}_{\text {proc }}$ is the Processing Delay, $\mathrm{L}$ is the packet Length, $\mathrm{R}_{\mathrm{b}}$ is the transmission data rate

- Energy per packet: In general energy is consumed when packets are sent to nodes It is defined as transmission energy par packet needed to transmit a single packet via wireless link.

Energy Packet $=P_{S} . T_{S}$

Where, Ps is the transmission power, Ts is the transmission time

- Received signal strength (RSSI): RSSI is a measure of how strong the most recent signal was when it reached its destination. Signal strength is also affected by the distance between the devices and any other objects that are directly placed between them [13].

$10 * \log P_{r}=10 * \log P_{S}-10 n * \log d$

' $\mathrm{P}_{\mathrm{r}}$ ' is the received power, ' $\mathrm{P}_{\mathrm{s}}$ ' is the transmitted power, ' $\mathrm{n}$ ' is the signal propagation and ' $\mathrm{d}$ ' is the distance between sender \& receiver. 


\title{
International Journal of Advanced Research in Electrical, Electronics and Instrumentation Engineering
}

\author{
(An ISO 3297: 2007 Certified Organization)
}

\section{Vol. 3, Issue 9, September 2014}

Delay Fig. 4 shows the delay per packet length. Delay is calculated with respect to packet length. The analysis shows that delay increases as the packet length is increased. Delay in Enocean standard is less as compared to Zigbee standard.

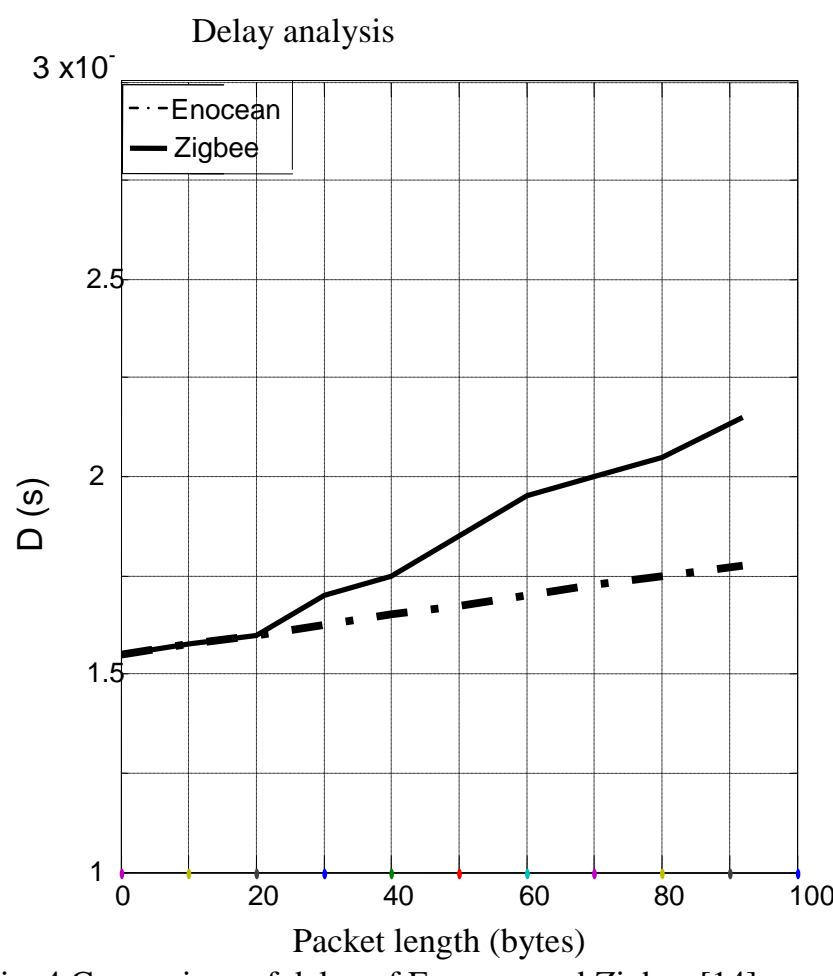

Fig. 4 Comparison of delay of Enocean and Zigbee [14].

In Fig. 4 Enocean delay is less as compared to Zigbee. As we know delay is dependent upon propagation delay and processing time according to equation 1. Processing time for Enocean is small as compared to Zigbee because Enocean transmission/ACK cycle is very small as compared to Zigbee. Therefore propagation delay also increases. Which is defined as total time taken after full message has been sent from sender until it has reached the receiving node. The values to support the result are shown in table 1

\begin{tabular}{|l|l|l|}
\hline Packet length(bytes) & (Zigbee) Delay $(\mathrm{msec})$ & $($ Enocean) Delay $(\mathrm{msec})$ \\
\hline 20 & 1.6 & 1.6 \\
\hline 40 & 1.7 & 1.6 \\
\hline 60 & 1.97 & 1.69 \\
\hline 80 & 2.1 & 1.7 \\
\hline
\end{tabular}

Table 1 Value of delay versus packet length

It is clear from the Table 1 that delay is gradually increases for both standards like at 20 packets delay is minimum i.e. 1.6 for both standards whereas at 80 packets delay is maximum i.e. 2.7 for Zigbee and 1.7 for Enocean. Another reason for increase in delay is collisions. In Zigbee there are more collisions due to high frequency.

Energy per packet Fig. 5 shows the comparison of Energy per packet with respect to links between different rooms. There are five rooms in which F1 and F2 represents the link between first and second room, F2 and F3 represents the link between second and third room and so on. F6 link represents the hallway in which there are more walls and furniture. This graph shows that Enocean consumes less energy as compared to Zigbee. 


\title{
International Journal of Advanced Research in Electrical, Electronics and Instrumentation Engineering
}

\author{
(An ISO 3297: 2007 Certified Organization)
}

\section{Vol. 3, Issue 9, September 2014}

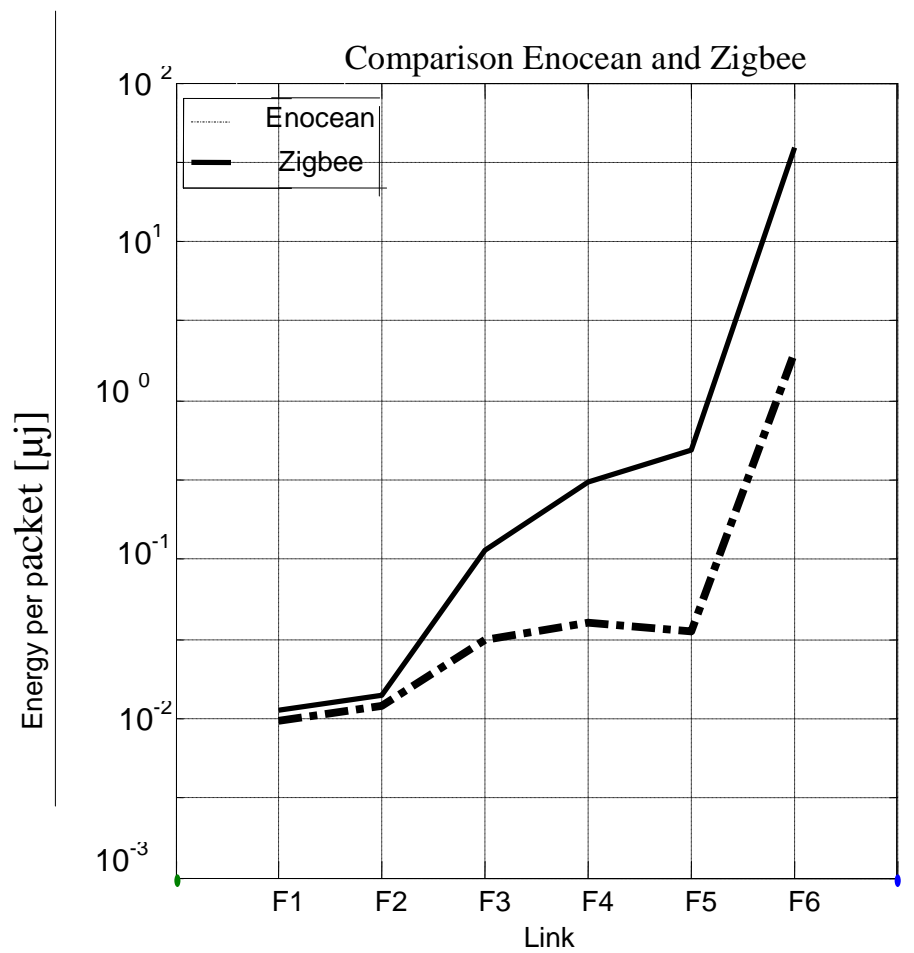

Fig. 5 Comparison of energy per packet for Enocean and Zigbee [1].

From this graph we see that Enocean consumes less energy as compared to Zigbee because according to equation 3 energy per packet depends upon the transmission time and transmission power. Enocean technology is capable of using less power than Zigbee. Data packets are relatively small (a typical data packet is 4 bytes)and it is transmitted at much lower frequency $(315 \mathrm{MHz}$ or $868 \mathrm{MHz}$ ) than Zigbee. Another reason is that Zigbee radio takes 15 30 milliseconds to complete a transmission/ACK cycle whereas Enocean takes milliseconds by which collision probability increases. Another reason is that due to high frequency there are more collisions by which delay increases. Therefore Zigbee consumes more energy to transmit data from sender to receiver. Further this comparison is clear by the table 2 in which values are given for energy at each link.

\begin{tabular}{|l|l|l|}
\hline link & $($ Zigbee $)$ Energy $(\mu \mathrm{j})$ & $($ Enocean $)$ Energy $(\mu \mathrm{j})$ \\
\hline F1 & $10^{-1.9}$ & $10^{-2}$ \\
\hline F2 & $10^{-1.8}$ & $10^{-1.9}$ \\
\hline F3 & $10^{-1}$ & $10^{-1.5}$ \\
\hline F4 & $10^{-0.5}$ & $10^{-1.4}$ \\
\hline F5 & $10^{-0.6}$ & $10^{-1.45}$ \\
\hline F6 & $10^{-0.5}$ & $10^{-0.4}$ \\
\hline
\end{tabular}

Table 2 Value of energy per packet versus links

Table 2 shows that less energy is consumed at F1 link for both standards because here number of walls are less whereas at F6 more energy is consumed because here number of walls are more as compared to other rooms and more furniture is placed

\section{Received Signal Strength Indicator (RSSI):}

Fig. 6 shows the comparison of RSSI value of Enocean and Zigbee and distance between different rooms [2]. Results show that Enocean has better RSSI value as compared to Zigbee. Fig. 6 shows that Enocean value is less negative than Zigbee so it's better as compared to Zigbee. As we know construction materials and obstructions have a significant 


\title{
International Journal of Advanced Research in Electrical, Electronics and Instrumentation Engineering
}

\author{
(An ISO 3297: 2007 Certified Organization)
}

\section{Vol. 3, Issue 9, September 2014}

effect on the reliability and strength of a wireless link. RSSI depends upon the strength of wireless link. Weaker signals have less received signal strength on receiver side. Received signal strength is also dependent upon the penetration power. Like for plaster walls/drywalls Enocean typically ranges $30 \mathrm{~m}$ through 5 walls whereas for brick walls its range is $20 \mathrm{~m}$ through 3 walls. This comparison can easily be understood by the table 3 in which values are given for RSSI w.r.t. distance.

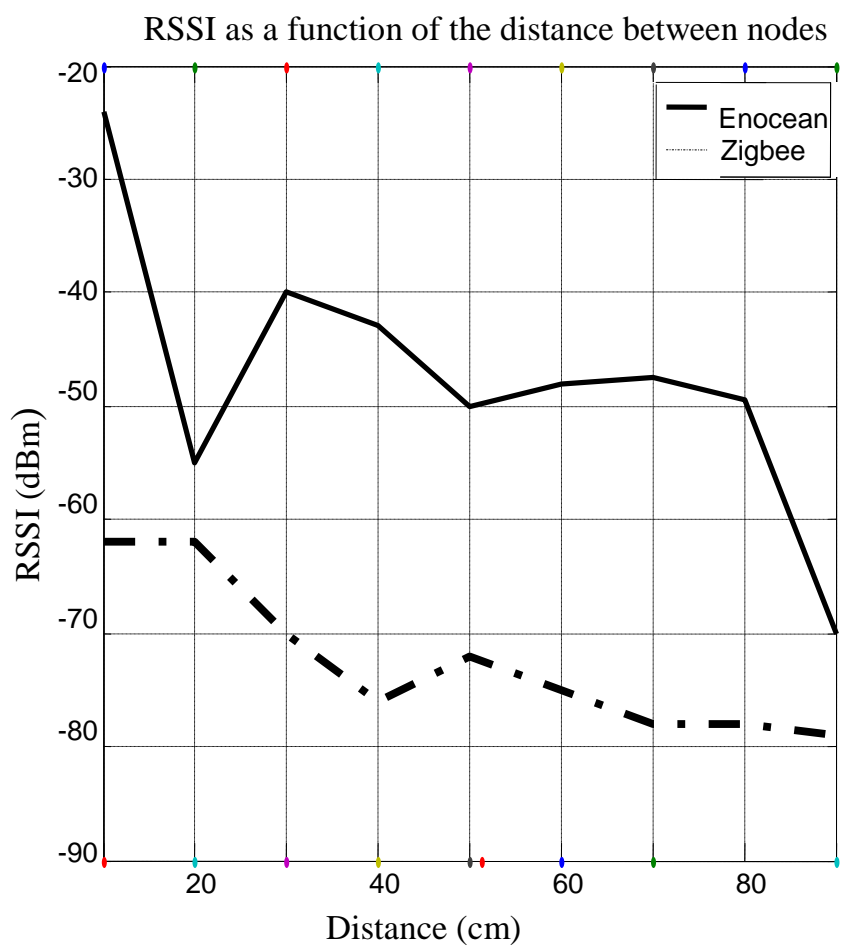

Fig. 6 Comparative RSSI.

\begin{tabular}{|l|l|l|}
\hline Packet length(bytes) & (Zigbee) RSSI (dbw) & (Enocean) RSSI (dbw) \\
\hline 20 & -61 & -55 \\
\hline 40 & -76 & -42 \\
\hline 60 & -75 & -48 \\
\hline 80 & -78 & -50 \\
\hline
\end{tabular}

Table 3 Value of RSSI versus Distance.

\section{CONCLUSION}

Wireless technologies have become very popular in both home and commercial network applications. The use of wireless technologies offers distinctive advantage in the field of home and building automation (HBA). Installations costs are significantly reduced since wiring is not required. So, Wireless is the best solution to control homes automatically. For this reason two wireless standards Zigbee and Enocean are choose to control the temperature of the house. For which first the model of Zigbee and Enocean in Matlab/Simulink is being created. Then these two models are embedded in house to control the temperature of house through temperature sensor which is controlled by the Zigbee and Enocean standard separately. Zigbee is a Light weight IEEE 802.15.4 standard based technology for low power consumption. Whereas Enocean is recently published as a IEC standard which operates with ultra low power. To investigate the performance of these standards three parameters are considered i.e. delay, energy per packet, and received signal strength (RSSI). Results show that, Enocean delay is less as compared to Zigbee. Average end to end delay is dependent upon propagation delay which is also dependent upon propagation speed. Therefore Enocean delay 


\title{
International Journal of Advanced Research in Electrical, Electronics and Instrumentation Engineering
}

\author{
(An ISO 3297: 2007 Certified Organization)
}

\section{Vol. 3, Issue 9, September 2014}

is less. Energy per packet is dependent upon the transmission power which is very low for Enocean. Last parameter is received signal strength which is dependent upon the distance between nodes and it is also affected by the objects between the devices. Distance is same for both standards but Enocean has more penetration power through the walls. Therefore Enocean has better RSSI as compared to Zigbee. It is therefore concluded here that Enocean is better than Zigbee. For future work photoelectric sensor, occupancy sensor can be used to control the light of the house. Security can also be provided through smoke detect sensors or water level sensors. Several other parameters such as throughput, signal to noise ratio, jitter can be used to investigate the performance of these standards to give the better solution to automate home with less cost and high reliability.

\section{REFERENCES}

1. Gomez, C.and paradells, J., "Wireless home automation networks: A survey of architectures and technologies", Comm. Mag. Vol. 48, pp. 92$101,2010$.

2. Trichakis, D., Chousidis, C., Rigakis, I., Antonidakis, E., "Power line Network Automation over IP", International Conference on Telecommunications and Multimedia (TEMU), vol. 978, pp. 239-244, 2012

3. Han, D. M. and Lim, J.H., "Smart home energy management system using IEEE 802.15 .4 and Zigbee," IEEE Trans. Consumer Electron. vol. 56, Aug. 2010.

4. Han, D.M. and Lim, J.H., "Design and implementation of smart home energy management systems based on Zigbee", IEEE Trans. Consum.Electron., vol. 56, no. 3, pp. 1417-1425, Aug. 2010.

5. Suryadeva, N.K., Gaddam, A., Rayudu, R. K. and Mukhopadhyay, S.C., "Wireless Sensor Networks based Safe Home to Care Elderly People: Behavior Detection", Sensors and Actuators, vol. 186, pp. 277-283, 2012.

6. Lee, J.S., Su, Y. W. and Shen, C. C., “A Comparative study of Wireless Protocols: Bluetooth, UWB, Zigbee, and Wi-Fi”, Industrial Electronics Society, 33rd Annual conference of the IEEE, Nov. 2007.

7. Tian, B., Han, S., Liu, L., Khadem and S., Parvin, S., "Towards enhanced key management in multi-phase ZigBee network architecture" Computer Communications, vol. 35, pp. 579-588, 2012.

8. Enocean literature, www.enocean.com/...pdf.../...WP_EnOcean_Technology_en_jul11.pdf.

9. Enocean Literature, Enocean Dolphin EO3000I API, 2.0 editions, Feb. 2010.

10. Ferrari, G., Medagliani, P., Piazza, S. D. and Martal, M. "Wireless Networks: "Performance analysis in Indoor Scenarios", EURASIP Journal on Wireless Communications and Networking, vol. 2007, 2007.

11. Zigbee model from MATHWORKS, www.mathworks.in/matlab central/fileexchange/36258-modulation-demodulation-in-zigbee.

12. Alam, M. R., Reaz, M. B. I. and Ali, M. A. M.," A Review of Smart Homes-Past, Present, and Future", IEEE transactions on systems, man, and cybernetics — part c: applications and reviews, pp. 1094-6977, IEEE 2012.

13. Gautam, D. R., Sharma, S. and Sahu, S., "Enhanced Transmission Power Control Mechanism based on RSSI for MANET", vol. 28, no. 1, pp. 25-30, August 2011.

14. Langhammer, N.and Kays, R., "Performance evaluation of wireless home automation networks in indoor scenarios", Transactions on smart grid, 10, IEEE July 2012. 